\title{
Preparation of Biodegradable Oligo(lactide)s-Grafted Dextran Nanogels for Efficient Drug Delivery by Controlling Intracellular Traffic
}

\author{
Yuichi Ohya ${ }^{1,2, *(\mathbb{D})}$, Akihiro Takahashi ${ }^{2}$ and Akinori Kuzuya ${ }^{1,2}$ (1) \\ 1 Department of Chemistry and Materials Engineering, Faculty of Chemistry, Materials and Bioengineering, \\ Kansai University, 3-3-35 Yamate, Suita, Osaka 564-8680, Japan; kuzuya@kansai-u.ac.jp \\ 2 Organization for Research and Development of Innovative Science and Technology (ORDIST), \\ Kansai University, 3-3-35 Yamate, Suita, Osaka 564-8680, Japan; akki.taka4@gmail.com \\ * Correspondence: yohya@kansai-u.ac.jp
}

Received: 13 April 2018; Accepted: 27 May 2018; Published: 30 May 2018

\begin{abstract}
Nanogels, nanometer-sized hydrogel particles, have great potential as drug delivery carriers. To achieve effective drug delivery to the active sites in a cell, control of intracellular traffic is important. In this study, we prepared nanogels composed of dextran with oligolactide (OLA) chains attached via disulfide bonds (Dex-g-SS-OLA) that collapse under the reductive conditions of the cytosol to achieve efficient drug delivery. In addition, we introduced galactose (Gal) residues on the nanogels, to enhance cellular uptake by receptor-mediated endocytosis, and secondary oligo-amine (tetraethylenepentamine) groups, to aid in escape from endosomes via proton sponge effects. The obtained Dex-g-SS-OLA with attached Gal residues and tetraethylenepentamine $\left(\mathrm{EI}_{4}\right)$ groups, $\mathrm{EI}_{4} / \mathrm{Gal}$-Dex-g-SS-OLA, formed a nanogel with a hydrodynamic diameter of ca. $203 \mathrm{~nm}$ in phosphate-buffered solution. The collapse of the $\mathrm{EI}_{4} / \mathrm{Gal}$-Dex-g-SS-OLA nanogels under reductive conditions was confirmed by a decrease in the hydrodynamic diameter in the presence of reductive agents. The specific uptake of the hydrogels into HepG2 cells and their intercellular behavior were investigated by flow cytometry and confocal laser scanning microscopy using fluorescence dye-labeled nanogels. Escape from the endosome and subsequent collapse in the cytosol of the $\mathrm{EI}_{4} /$ Gal-Dex-g-SS-OLA were observed. These biodegradable nanogels that collapse under reductive conditions in the cytosol should have great potential as efficient drug carriers in, for example, cancer chemotherapy.
\end{abstract}

Keywords: biodegradable nanogel; disulfide bond; drug delivery; cellular traffic

\section{Introduction}

Nanogels are nanometer-sized hydrogel particles (diameter $<1 \mu \mathrm{m}$ ) composed of threedimensional networks of physically (or chemically) cross-linked polymer chains. Such nanogels have attracted much attention over the last decade due to their potential for applications in biomedical fields, such as for drug delivery systems (DDS) and bioimaging [1-13]. A physically cross-linked nanogel was first reported by Akiyoshi and coworkers in 1997 [14]. They reported that hydrophobized polysaccharides can form physically cross-linked nanogels in aqueous solution and entrap both hydrophobic and hydrophilic molecules, such as proteins [4]. Physical entrapment and the release behavior of proteins into and from nanogels have been investigated for applications as DDS carriers [9]. Biodegradability is one of the crucial factors for appropriate DDS carriers. Biodegradation offers several advantages, such as facilitation in releasing encapsulated molecules and excretion of the vehicles after release of the bioactive agents. We also reported biodegradable 
dextran-graft-oligo(L-lactide) (Dex-g-OLLA) nanogels and their stabilization by mixing with dextran-graft-oligo(D-lactide) (Dex-g-ODLA) via stereocomplex formation [15]. We succeeded in preparing protein-loaded Dex-g-OLLA nanogels using lysozyme as a model protein and found that the lysozyme-loaded nanogels showed sustained release of lysozyme for one week without denaturation in phosphate-buffered saline (PBS) at $37{ }^{\circ} \mathrm{C}$ [16].

To apply drug carriers in cancer chemotherapy, some additional functionalities are desired (Figure 1). First, enhanced permeability and retention (EPR) effects are necessary to accumulate the drug carriers in the highly vascular solid tumor tissue by passive targeting. Macromolecules with large hydrodynamic diameters or nanoparticles are more likely to be leaked from the neovascular tissue to accumulate in tumor tissue and are retained in the tissues due to the lack of lymph nodes $[17,18]$. Second, the carrier uptake into the cancer cells should occur via specific or non-specific interactions [19-21]. Endocytosis is one of the well-known cellular uptake processes, which only proceeds after the specific binding of ligands or antibodies against receptors or epitopes located on the cell membrane [21-23]. Third, after uptake into cancer cells, the carriers should escape from the endosomes into the cytosol. The proton sponge effect via protonation of secondary amine groups (like polyethyleneimine) on the carriers is one promising strategy for endosomal escape [24-26]. Finally, the carriers should collapse or be dissociated to release the drugs. Because the cytosol generally possesses reductive conditions, disulfide bonds have been utilized to release drugs in the cytosol, either by dissociation of the nanoparticles or by cleavage of the degradable bonds between carriers and drugs to expose the drugs to active sites or organelles in the target cells [27-29].

In this study, to achieve highly efficient cellular drug delivery to the cytosol by controlling intercellular traffic, we prepared nanogels composed of dextran (Dex) with oligolactide (OLA) chains attached via disulfide bonds to be collapsed under the reductive conditions of cytosol (Figure 1). In addition, the nanogels were equipped to enhance cellular uptake and escape from endosomes. The galactose (Gal) residue was chosen as a model ligand to enhance uptake into liver-derived cancer cells by receptor-mediated endocytosis [30,31]. Additionally, oligo-amine (tetraethylenepentamine, $\mathrm{EI}_{4}$ ) groups were attached to the nanogels to enhance escape from endosomes by proton sponge effects [24]. Finally, we investigated the interaction of the obtained nanogels with cells as well as their intracellular behavior.

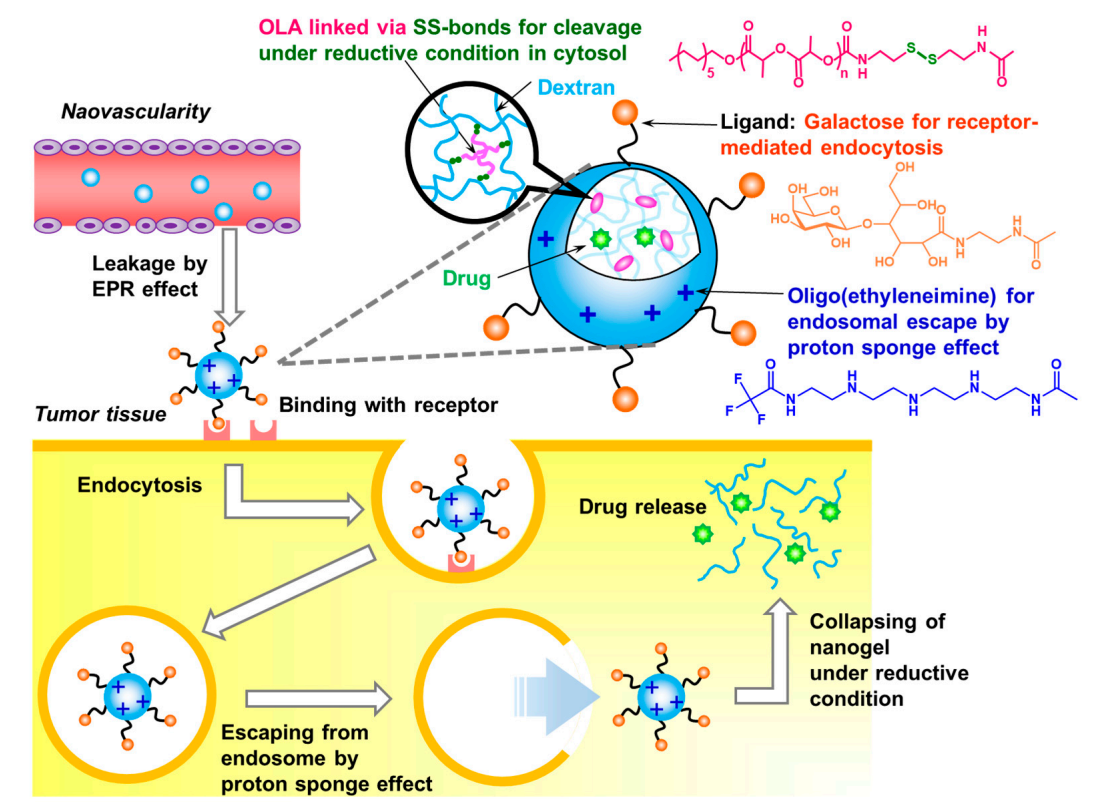

Figure 1. Schematic illustration for the concept of this work and the design of nanogel. EPR: enhanced permeability and retention, OLA: oligolactide, SS: disulfide. 


\section{Results and Discussion}

\subsection{Synthesis of Graft Copolymer}

OLA having a terminal primary amine group via a disulfide bond spacer (aminoethyl-disulfanylethyl-OLA, OLA-SS-NH$H_{2}$ ) was synthesized according to Scheme 1 (Supporting Information, Figure S1). Tetraethylenepentamine/galactose-modified dextran-graft-disulfanyl-ethyl-OLA (EI $4 / \mathrm{Gal}_{-} \mathrm{Dex}-\mathrm{g}$ SS-OLA) was prepared according to Scheme 2. Other control copolymers were also prepared by the variation of introduced components. The characterization of $\mathrm{EI}_{4} / \mathrm{Gal}-\mathrm{Dex}-\mathrm{g}-\mathrm{SS}-\mathrm{OLA}$ was carried out by ${ }^{1} \mathrm{H}$ nuclear magnetic resonance (NMR) spectroscopy (Figure S1), confirming the existence of Dex (3.05-3.90 and 4.83 ppm), OLA-SS units (1.08, 2.50, and 3.94 ppm), ethylene groups of $\mathrm{EI}_{4}$ (2.30-2.60 ppm), and Gal residues (2.67-2.80, 3.05-3.90, and $4.38 \mathrm{ppm})$. The amounts of OLA-SS units, $\mathrm{EI}_{4}$ groups, and Gal residues were calculated, by the integral ratios of the ${ }^{1} \mathrm{H} \mathrm{NMR}$ spectrum, to be 2.5, 35, and 30 molecules per Dex molecule, respectively. These values correspond to degrees of introduction of $0.9,13.2$, and $11.3 \mathrm{~mol} \%$ per sugar unit of Dex, respectively.

$$
\text { }
$$

Scheme 1. Synthesis of aminoethyl-disulfanyl-ethyl-OLA (hydrogen bromide salt) (OLA-SS- $\mathrm{NH}_{3}{ }^{+} \mathrm{Br}^{-}$). CDI: $N, N^{\prime}$-carbonyldiimidazole, DMAP: 4-(N,N-dimethylamino)-pyridine.

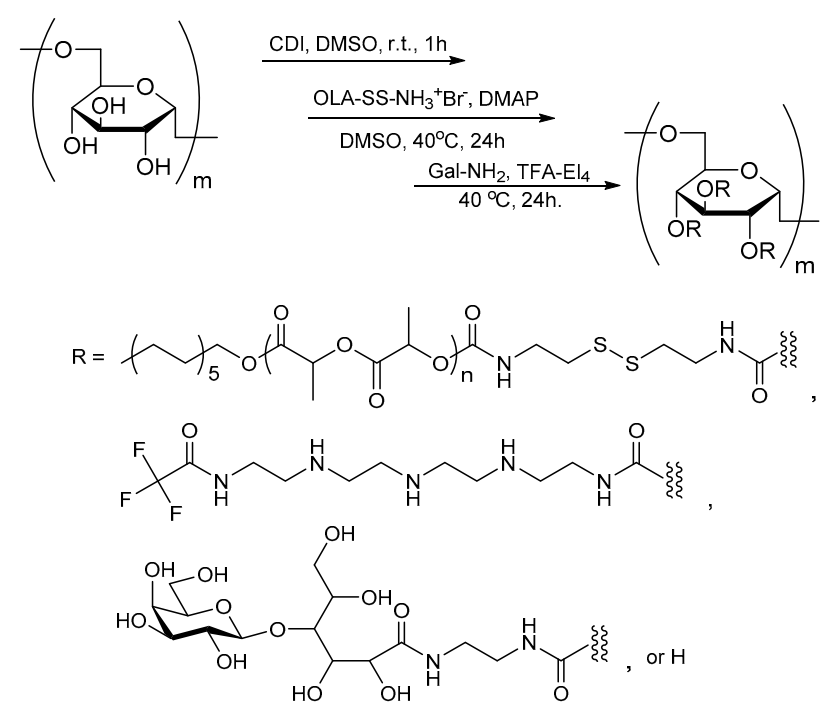

Scheme 2. Synthesis of tetraethylenepentamine/galactose-modified dextran-graft-disulfanyl-ethylOLA (EI ${ }_{4} /$ Gal-Dex-g-SS-OLA). DMSO: dimethylsulfoxide, TFA: trifluoroacetic acid.

\subsection{Characterization of Nanogels and Dissociation under Reductive Conditions}

Nanogels were prepared by a dialysis method. The hydrodynamic diameters of the nanogels were investigated with dynamic light scattering (DLS) measurements (Figure S3). The Z-averages of the hydrodynamic diameters $\left(D_{\mathrm{h}}\right)$ of Dex-g-OLA, Dex-g-SS-OLA, and EI $4 /$ Gal-Dex-g-SS-OLA nanogels are estimated to be 141, 141, and $203 \mathrm{~nm}$, respectively, and their top peak contributions 
are 199, 213, and $263 \mathrm{~nm}$. Dex-g-OLA and Dex-g-SS-OLA nanogels show similar $D_{\mathrm{h}}$, however the $\mathrm{EI}_{4} / \mathrm{Gal}-\mathrm{Dex}-\mathrm{g}$-SS-OLA nanogel shows a slightly larger $D_{\mathrm{h}}$ value, suggesting electrostatic repulsion and higher hydration of $\mathrm{EI}_{4}$ groups. The stability of the nanogel against dilution was evaluated through the critical aggregation concentration (CAC) value by a fluorescence probe method, using pyrene as the fluorescence probe (Figure S4). The CAC value of Dex-g-SS-OLA nanogels is estimated to be $5.9 \times 10^{-4} \mathrm{wt} \%$. This result is similar to the case of Dex-g-OLA nanogels previously reported [15].

The stability and dissociation behavior of the nanogels under reductive conditions were investigated by monitoring $D_{\mathrm{h}}$ after the addition of a reductive agent. Figure 2 shows the time course of the average $D_{\mathrm{h}}$ for the $\mathrm{EI}_{4} / \mathrm{Gal}$-Dex- $g$-SS-OLA nanogel monitored by DLS in the presence or absence of $10 \mathrm{mM}$ glutathione (GSH), as a reductive agent. Without GSH, the diameter of the $\mathrm{EI}_{4} / \mathrm{Gal}$-Dex-g-SS-OLA nanogel does not change. On the other hand, the hydrodynamic diameter decreases in the presence of GSH to be less than $10 \mathrm{~nm}$ in $1 \mathrm{~h}$. These results suggest that the disulfide bonds are cleaved in the presence of GSH as a reductive reagent, and the $\mathrm{EI}_{4} / \mathrm{Gal}_{-D e x}-\mathrm{g}$-SS-OLA nanogels collapse.

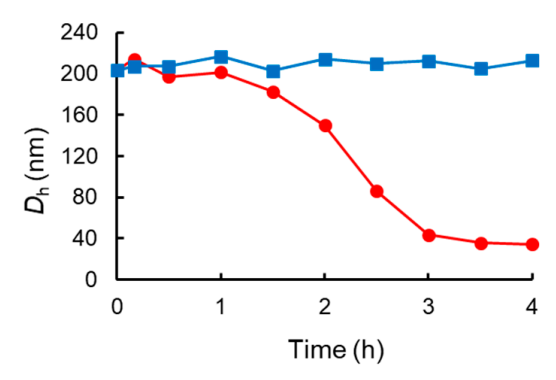

Figure 2. Time course of the Z-average of the hydrodynamic diameter $\left(D_{\mathrm{h}}\right)$ for the $\mathrm{EI}_{4} / \mathrm{Gal}$-Dex-g-SSOLA nanogel monitored by dynamic light scattering (DLS) in the presence (red circles) or absence (blue squares) of $10 \mathrm{mM}$ glutathione (GSH) in phosphate buffer (PB) solution.

The dissociation behavior of the $\mathrm{EI}_{4} / \mathrm{Gal}$-Dex- $g$-SS-OLA nanogels was investigated by other techniques. Figure 3 shows photographs of the original $\mathrm{EI}_{4} / \mathrm{Gal}-\mathrm{Dex}-\mathrm{g}$-SS-OLA nanogel solution and the nanogel solution after $24 \mathrm{~h}$ incubation in the presence of $10 \mathrm{mM}$ of dithiothreitol (DTT) as a reductive agent. The nanogel solution before incubation was slightly turbid and showed strong scattering by red laser beam irradiation, indicating dispersion of the particles at the hundreds-of-nanometers scale. On the other hand, the nanogel solution after $24 \mathrm{~h}$ incubation with DTT was a clear solution and almost no scattering was observed. These results indicate that the aggregates (nanogels) collapse into very small particles or soluble molecules without scattering. Furthermore, the time course of the fluorescence spectra for the $\mathrm{EI}_{4} / \mathrm{Gal}-\mathrm{Dex}-\mathrm{g}$-SS-OLA nanogels entrapping pyrene in the presence of $10 \mathrm{mM}$ DTT as a reductive agent is shown in Figure 4. After the addition of DTT, the fluorescence intensity of pyrene gradually decreases. These results indicate that the hydrophobic pyrene molecules are released from the nanogels and precipitate upon their collapse. The change in fluorescence spectra have a different tendency compared to the DLS measurement (Figure 2); the fluorescence decay took nearly $24 \mathrm{~h}$, while the $D_{\mathrm{h}}$ change in DLS measurements was complete after several hours. The DLS measurements reflect the direct dissociation of a larger nanogel into a partially collapsed nanogel having smaller (several tens of nanometers) diameter. However, the partially collapsed nanogels still have hydrophobic domains which can entrap pyrene molecules. This may be the reason that the complete release of pyrene took a longer time. From all of these results, $\mathrm{EI}_{4} / \mathrm{Gal}-\mathrm{Dex}-\mathrm{g}$-SS-OLA nanogels are presumably collapsed under the reductive conditions of the cytosol.

The cytotoxicity of $\mathrm{EI}_{4} /$ Gal-Dex-g-SS-OLA nanogels was investigated against L929 mouse fibroblast cells by the WST-8 assay (Figure 5). Sodium dodecyl sulfate (SDS, surfactant) was used as a positive control. No decrease in cell viability was observed for $\mathrm{EI}_{4} / \mathrm{Gal}$-Dex-g-SS-OLA nanogels below $1.0 \times 10^{-1} \mathrm{mg} / \mathrm{mL}$. 


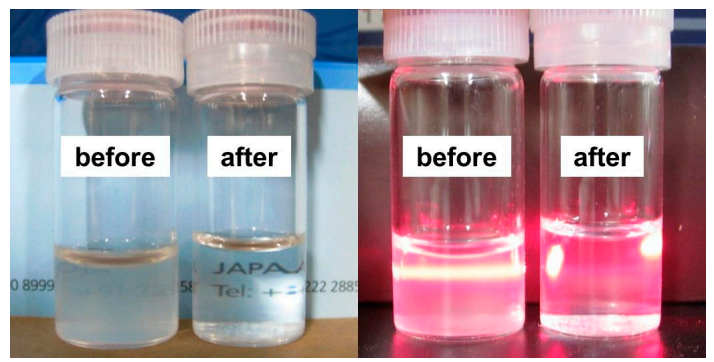

Figure 3. Photographs of $\mathrm{EI}_{4} /$ Gal-Dex-g-SS-OLA nanogel before and after $24 \mathrm{~h}$ incubation in the presence of $10 \mathrm{mM}$ dithiothreitol (DTT) in PB solution at $25^{\circ} \mathrm{C}$ with (right) and without (left) laser beam irradiation.

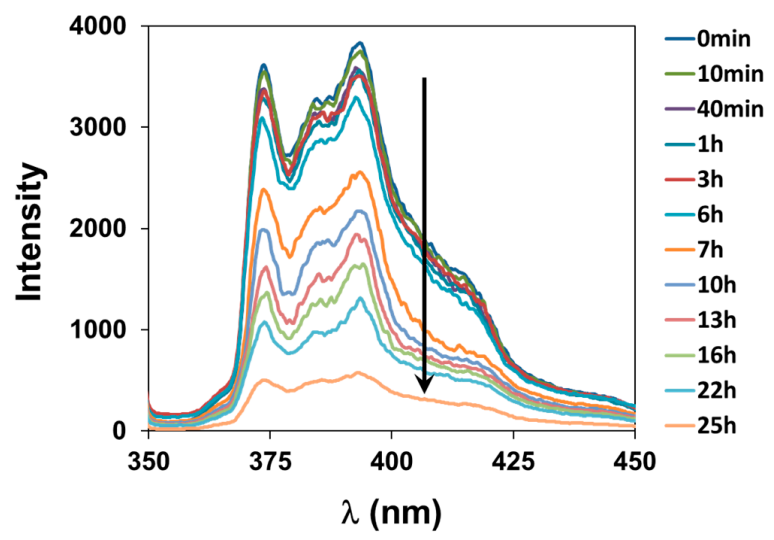

Figure 4. Time course of fluorescence spectra of pyrene entrapped in $\mathrm{EI}_{4} / \mathrm{Gal}-\mathrm{Dex}-\mathrm{g}$-SS-OLA nanogels after addition of $10 \mathrm{mM}$ DTT in PB solution at $25^{\circ} \mathrm{C}$. Black arrow means time course.

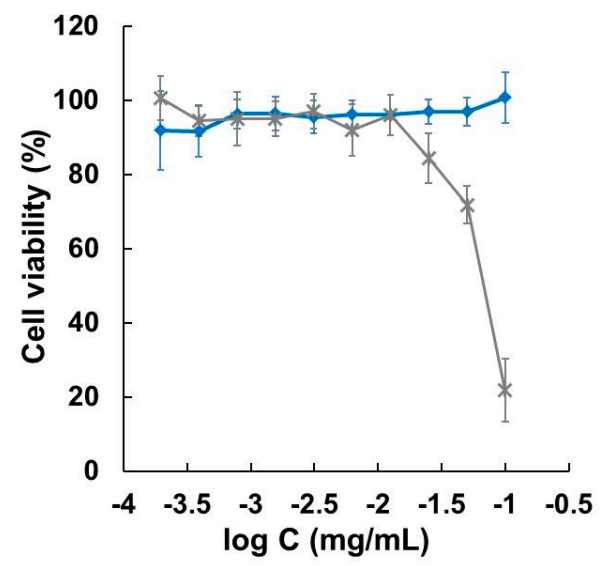

Figure 5. Viability of L929 cells after $24 \mathrm{~h}$ incubation as a function of concentrations of $(\downarrow)$ $\mathrm{EI}_{4} /$ Gal-Dex-g-SS-OLA nanogels and $(x)$ SDS.

\subsection{Cellular Uptake Behavior of EI $I_{4} /$ Gal-Dex-g-SS-OLA Nanogels}

To investigate the cellular uptake of the nanogels into HepG2 cells, flow cytometric analysis was carried out using fluorescein isothiocyanate isomer I (FITC)-labeled nanogels (Figure 6). A shift to the right (stronger fluorescence intensity at $525 \mathrm{~nm}$ ) in the cell distribution means that an uptake of FITC-labeled nanogels into HepG2 cells has occurred. After incubation with $\mathrm{EI}_{4} / \mathrm{Gal}_{-} \mathrm{Dex}-\mathrm{g}$-SS-OLA nanogels, a peak shift to the right is indeed observed compared with intact HepG2 and that incubated with Dex-g-SS-OLA nanogels without galactose residues. HepG2 cells incubated with 
$\mathrm{EI}_{4}$-Dex-g-SS-OLA nanogels with cationic $\mathrm{EI}_{4}$ groups but without galactose residues show a slight shift to the right, suggesting an electrostatic interaction of cationic groups of $\mathrm{EI}_{4}$-Dex-g-SS-OLA nanogels with $\mathrm{HeG} 2$ cells. Since $\mathrm{EI}_{4} / \mathrm{Gal}$-Dex-g-SS-OLA nanogels induce a larger shift to higher fluorescence intensity compared with $\mathrm{EI}_{4}$-Dex-g-SS-OLA nanogels, galactose receptor-mediated specific binding of $\mathrm{EI}_{4} /$ Gal-Dex-g-SS-OLA nanogels against HepG2 cells is suggested.

We performed confocal laser scanning microscopy (CLSM) of the HepG2 cells incubated with the nanogels to confirm locations of the nanogels in each cell and their collapse. The cells were incubated with FITC-labeled nanogels, $\mathrm{EI}_{4} / \mathrm{Gal}$-Dex-g-SS-OLA, EI $/$ /Gal-Dex-g-OLA (without -SSbonds), Gal-Dex-g-SS-OLA (without $\mathrm{EI}_{4}$ ), and $\mathrm{EI}_{4}$-Dex-g-SS-OLA (without Gal). The results are shown in Figures 7 and 8. The nanogels and lysosomes are observed as green fluorescence by FITC and red fluorescence by LysoTracker RED, respectively. Figure 7 shows CLSM images of HepG2 cells with various nanogels after $5 \mathrm{~min}$ incubation at $37^{\circ} \mathrm{C}$. EI $\mathrm{E}_{4} / \mathrm{Gal}$-Dex-g-SS-OLA, Gal-Dex-g-SS-OLA, and $\mathrm{EI}_{4} / \mathrm{Gal}-\mathrm{Dex}-\mathrm{g}$-OLA nanogels with $\mathrm{Gal}$ residues show relatively strong green fluorescence at the periphery of the cells, although $\mathrm{EI}_{4}$-Dex-g-SS-OLA do not show very strong fluorescence. These results suggest that nanogels with Gal residues bind to the HepG2 cells by specific galactose receptor mediated interactions, and the electrostatic interaction between cationic $\mathrm{EI}_{4}$ groups on the nanogels and the cells is not dominant. Overlap of red fluorescence of LysoTracker RED and green fluorescence of FITC is not so obvious for all nanogels. This may be because endocytosis is not completed in $5 \mathrm{~min}$, and the observed nanogels exist on the cell surfaces. The cells were further incubated at $37{ }^{\circ} \mathrm{C}$ for $5 \mathrm{~h}$ (Figure 8). In Figure 8c, the green and red fluorescence spots are observed at almost the same positions for Gal-Dex-g-SS-OLA nanogels without $\mathrm{EI}_{4}$ groups, suggesting that the Gal-Dex-g-SS-OLA nanogels uptaken by receptor-mediated endocytosis were localized at endosomes (or lysosomes). In Figure 8b, the green and red fluorescence spots are observed at different positions for $\mathrm{EI}_{4} / \mathrm{Gal}$-Dex-g-OLA nanogels without SS linkages, suggesting that the $\mathrm{EI}_{4} / \mathrm{Gal}$-Dex-g-OLA nanogels can escape from the lysosome. However, the green fluorescence is observed as spots suggesting the nanogels retain their particle shapes and do not diffuse very much in the cytosol. On the other hand, in Figure 8a, the green fluorescence is spread over a wider area in the cytosol for $\mathrm{EI}_{4} / \mathrm{Gal}$-Dex-g-SS-OLA nanogels. These results suggest that the $\mathrm{EI}_{4} / \mathrm{Gal}$-Dex-g-SS-OLA nanogels collapse and the FITC-labeled copolymer can diffuse in the cytosol. Therefore, the introduction of $\mathrm{EI}_{4}$ as a cationic group is effective for escape from the endosomes, and the -SS- group can be cleaved under the reductive conditions of the cytosol to collapse $\mathrm{EI}_{4} /$ Gal-Dex-g-SS-OLA nanogels.

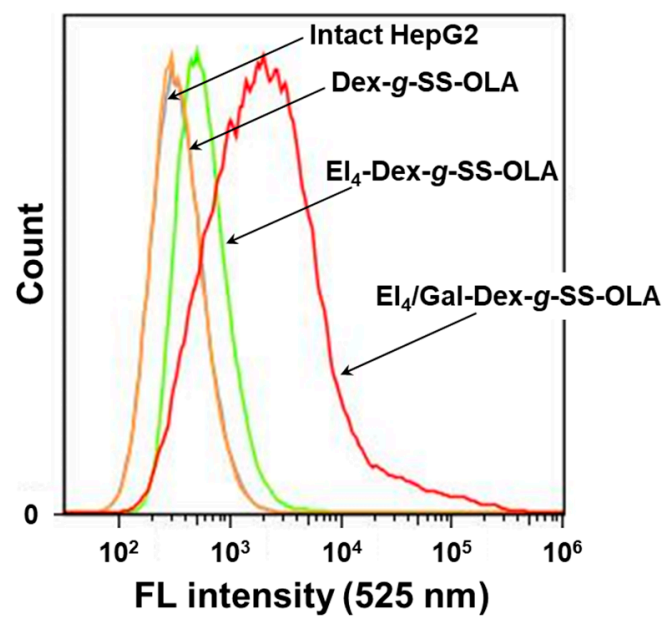

Figure 6. Results of flow cytometric analysis for HepG2 cells incubated with fluorescein isothiocyanate isomer I (FITC)-labeled nanogels for $4 \mathrm{~h}$. Intact HepG2 cells (grey, almost overlapped with orange), Dex-g-SS-OLA (orange), EI 4 -Dex-g-SS-OLA (light green), EI 4 /Gal-Dex-g-SS-OLA nanogels (red). FL: fluorescence. 


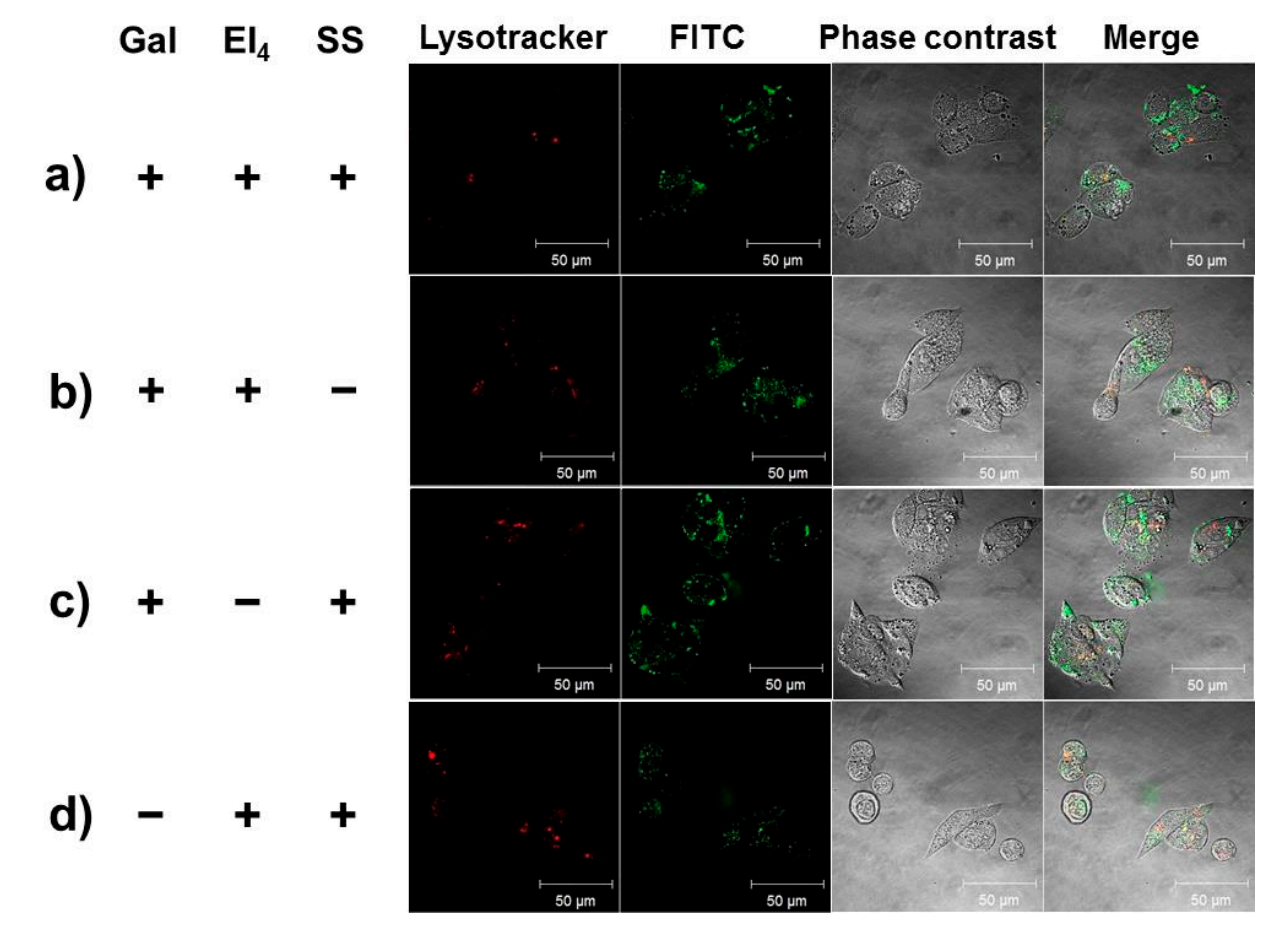

Figure 7. Confocal laser scanning microscopy (CLSM) images of HepG2 cells with various FITC-labeled nanogels and LysoTracker RED after $5 \mathrm{~min}$ incubation at $37^{\circ} \mathrm{C}$. (a) $\mathrm{EI}_{4} / \mathrm{Gal}^{-D e x-g-S S-O L A}$, (b) $\mathrm{EI}_{4} /$ Gal-Dex-g-OLA, (c) Gal-Dex-g-SS-OLA, and (d) EI ${ }_{4}$-Dex-g-SS-OLA. Scale bar: $50 \mu \mathrm{m}$.

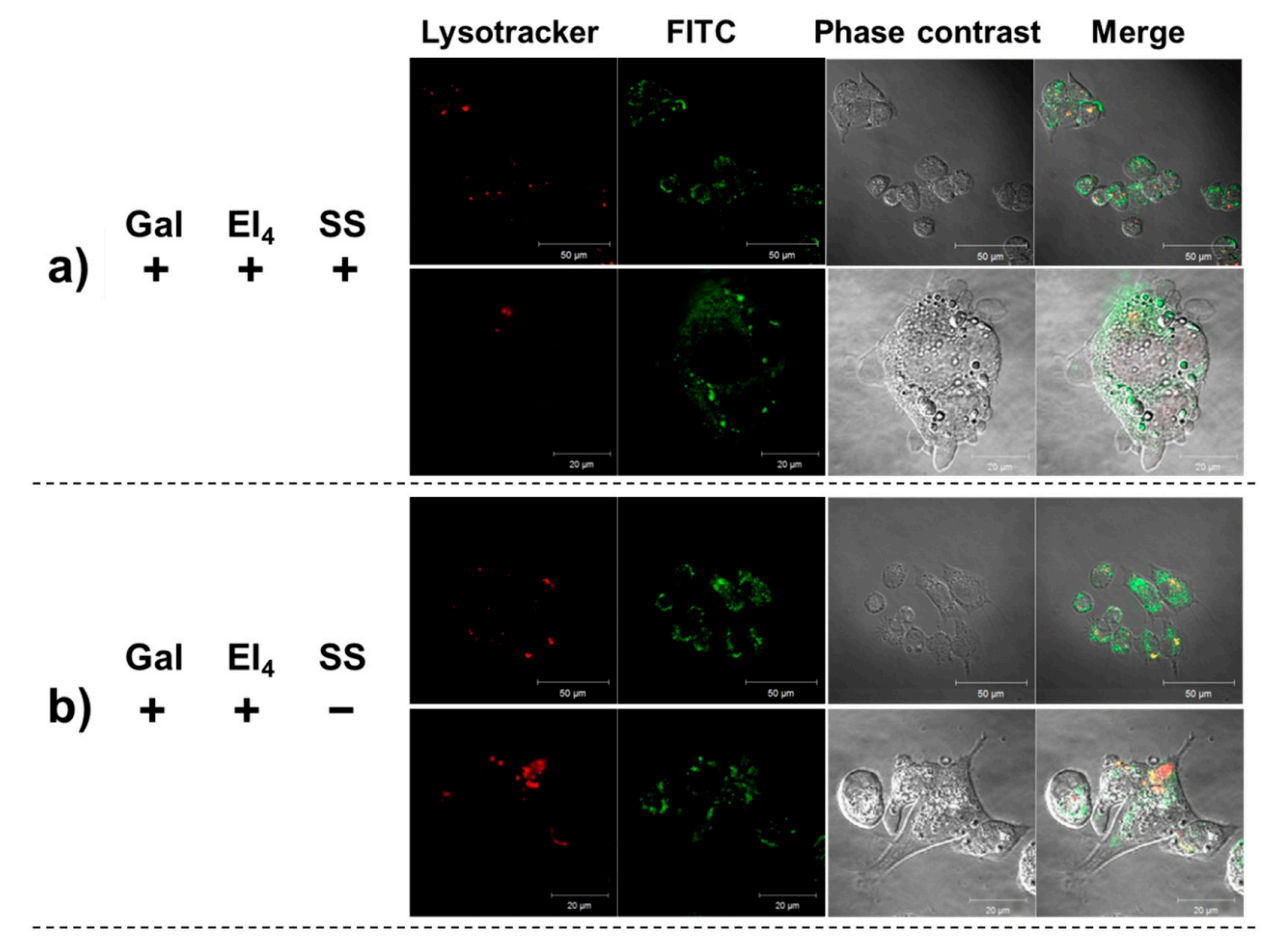

Figure 8. Cont. 


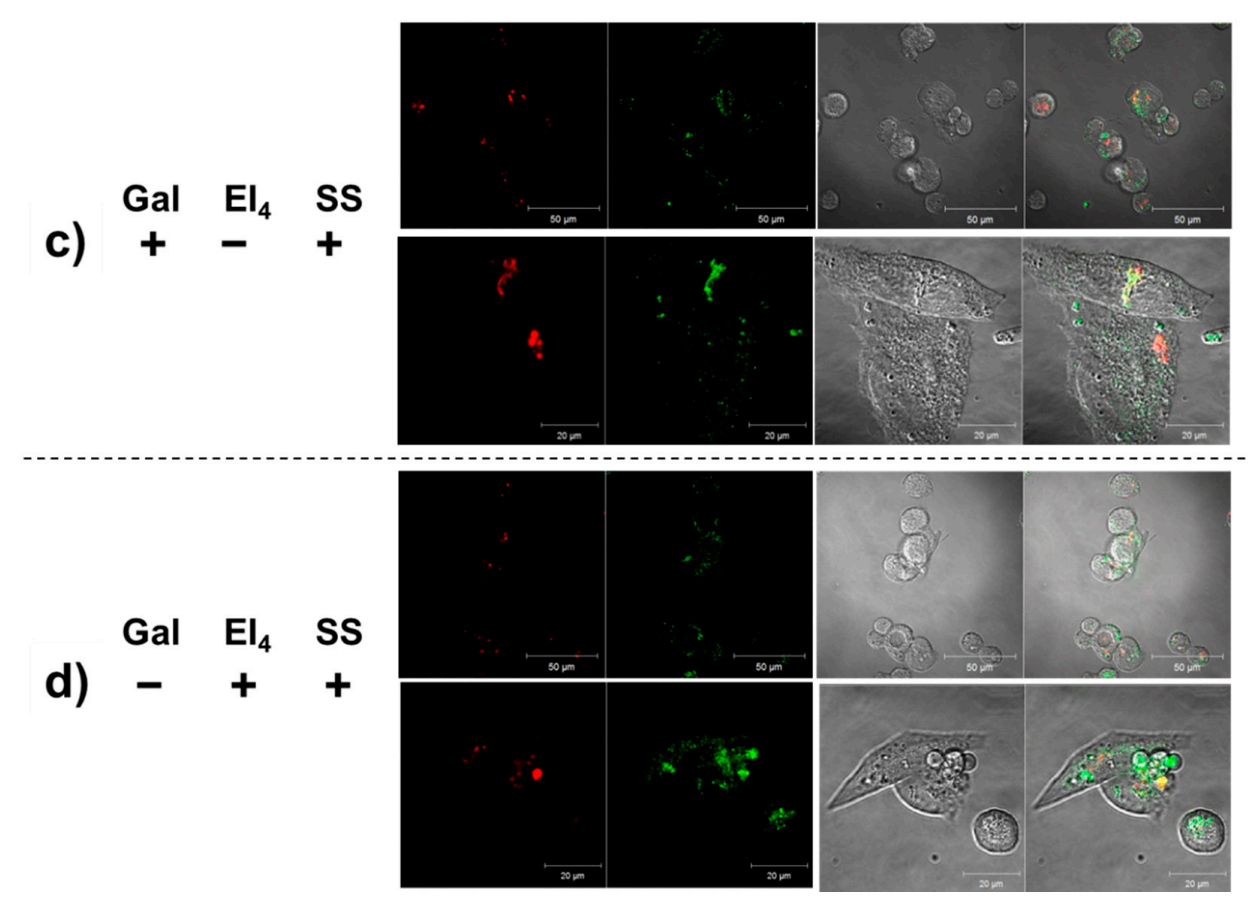

Figure 8. CLSM images of HepG2 cells with various FITC-labeled nanogels and LysoTracker RED after $5 \mathrm{~h}$ incubation at $37^{\circ} \mathrm{C}$. (a) EI 4 /Gal-Dex-g-SS-OLA, (b) EI 4 /Gal-Dex-g-OLA, (c) Gal-Dex-g-SS-OLA, and (d) $\mathrm{EI}_{4}$-Dex-g-SS-OLA. Scale bar: $50 \mu \mathrm{m}$ (top row of each panel) and $20 \mu \mathrm{m}$ (bottom row of each panel).

\section{Materials and Methods}

\subsection{Materials}

L-Lactide was purchased from Musashino Chemical Laboratory, Ltd. (Tokyo, Japan) and used without further treatment. Dextran (Dex) $\left(M_{\mathrm{W}}=43,000\right)$, fluorescein isothiocyanate isomer I (FITC) and ethylenediamine were purchased from Sigma-Aldrich (St. Louis, MO, USA). $N, N^{\prime}$-Carbonyldiimidazole (CDI), 4-(N,N-dimethylamino)-pyridine (DMAP), di-tert-butyl dicarbonate, cystamine dihydrochloride, dithiothreitol (DTT), and lactose were purchased from Wako Pure Chemical Ind., Ltd. (Osaka, Japan). Ethyl trifluoroacetate, tetraethylenepentamine $\left(\mathrm{EI}_{4}\right)$, and glutathione (GSH) were purchased from Tokyo Chemical Industry Co., Ltd. (Tokyo, Japan). LysoTracker RED was obtained from Thermo Fisher Scientific K.K. (Tokyo, Japan). Other reagents were commercial grade and used without further purification. Oligo(L-lactide) (OLA) (Mw = $3070 \mathrm{Da}$, degree of polymerization $=20$ ) was prepared by the usual ring-opening polymerization of L-lactide using tin(2-ethylhexanoate) $\left(\mathrm{Sn}(\mathrm{Oct})_{2}\right)$ as a catalyst and 1-dodecanol as an initiator, according to the literature [32]. $N$-Lactonoyl-1,4-ethylenediamine $\left(\mathrm{Gal}-\mathrm{NH}_{2}\right)$ and mono- $t$-butoxycarbonyl-cystamine (Boc-cystamine) were prepared by the methods reported previously with minor modification $[33,34]$. N-[2-[[2-[[2-[(2-Aminoethyl)amino]ethyl]amino]ethyl]amino]ethyl]-2,2,2-trifluoroacetamide (TFA-EI 4$)$ was synthesized from ethyl trifluoroacetate and $\mathrm{EI}_{4}$ by a previously reported method [35]. Fetal calf serum (FCS) was obtained from Thermo Fisher Scientific (Waltham, MA, USA). Eagle's minimum essential medium (E-MEM) and Dulbecco's minimum essential medium (D-MEM) were purchased from Nissui Pharmaceutical Co. (Tokyo, Japan).

\subsection{Measurements}

${ }^{1} \mathrm{H}$ NMR spectra were recorded on a JNM-GSX-400 (JEOL, Tokyo, Japan, $400 \mathrm{MHz}$ ) nuclear magnetic resonance spectrometer using deuterated solvent. The chemical shifts were calibrated against tetramethylsilane (TMS) and/or solvent signal. The number average molecular weights (Mn) 
and polydispersity indices $\left(M_{\mathrm{W}} / \mathrm{Mn}\right)$ of OLA were determined by size exclusion chromatography (SEC) (column: TSKgel Multipore HXLM $\times 2$; detector: RI). The measurements were performed using dimethylformamide (DMF) as an eluent at a flow rate of $1.0 \mathrm{~mL} / \mathrm{min}$ at $40{ }^{\circ} \mathrm{C}$ and a series of polystyrenes were used as standards. Hydrodynamic diameters of nanogels were measured by DLS (Zetasizer nano Z ZEN 2600, Malvern Instruments, Ltd., Malvern, UK). Fluorescence emission spectra of pyrene in the presence of nanogels were recorded by a fluorescence spectrophotometer (Shimadzu UV-2400PC, SIMADZU Co., Kyoto, Japan) using the excitation wavelength of $335 \mathrm{~nm}$ at room temperature (rt) in $\mathrm{PB}$ (phosphate buffer) solution $(50 \mathrm{mM}, \mathrm{pH}$ 7.4). The critical aggregation concentration (CAC) of the nanogels in PB solution were determined using pyrene as a fluorescence probe. Fluorescence intensities of $I_{1}$ and $I_{3}$ were measured at the wavelengths of 373 and $383 \mathrm{~nm}$, corresponding to the first and third vibronic bands. The CAC can be obtained from the flexion point in the $I_{1} / I_{3}$ ratio vs. concentration plots.

\subsection{Synthesis of Aminoethyl-Disulfanyl-Ethyl-OLA (OLA-SS-NH $\left.\mathrm{N}_{2}\right)$}

Aminoethyl-disulfanyl-ethyl-OLA (OLA-SS- $\mathrm{NH}_{2}$ ) was synthesized according to Scheme 1. OLA $(1.00 \mathrm{~g}, 326 \mu \mathrm{mol})$ was dissolved in $3 \mathrm{~mL}$ of anhydrous dichloromethane with CDI (159 mg, $977 \mu \mathrm{mol})$, and stirred at $\mathrm{rt}$ for $24 \mathrm{~h}$. The reaction mixture was poured into ethanol $/ n$-hexane $(3 / 7, v / v)$ and washed three times. The precipitate was dried in vacuo at $\mathrm{rt}$ to give a white powder of activated OLA (CI-OLA) (993 mg, yield 96\%). The CI-OLA (993 mg, $314 \mu \mathrm{mol})$, Boc-cystamine (152 mg, $942 \mu \mathrm{mol}$ ), and DMAP $(25 \mathrm{mg}, 314 \mu \mathrm{mol})$ were dissolved in anhydrous dichloromethane, and stirred at $\mathrm{rt}$ for $15 \mathrm{~h}$. The obtained product was precipitated by ethanol $/ n$-hexane $(3 / 7, v / v)$, washed three times, and dried in vacuo at rt to give a white powder of Boc-cystamine-OLA (921 mg, yield 87\%). Boc-cystamine-OLA (921 mg, $275 \mu \mathrm{mol})$ was dissolved in $3 \mathrm{~mL}$ of chloroform while cooling at $0{ }^{\circ} \mathrm{C}$. $\mathrm{HBr} / \mathrm{AcOH}(25 \%)$ $(343 \mu \mathrm{L}, 1.38 \mathrm{mmol})$ was added to remove the Boc group and the mixture was stirred at $0{ }^{\circ} \mathrm{C}$ for $4 \mathrm{~min}$. The reaction mixture was poured into diethylether to precipitate, washed three times, and dried in vacuum at $\mathrm{rt}$ to give a white powder of aminoethyl-disulfanyl-ethyl-OLA (OLA-SS-NH$\left.{ }_{2}\right)(884 \mathrm{mg}$, yield $98 \%$ ).

${ }^{1} \mathrm{H}$ NMR $\left(400 \mathrm{MHz}, \mathrm{CDCl}_{3}\right): \delta=0.89\left(\mathrm{t}, \mathrm{CH}_{3} \mathrm{CH}_{2}-\right), 1.20-1.38\left[\mathrm{~m}, 20 \mathrm{H}, \mathrm{CH}_{3}\left(\mathrm{CH}_{2}\right)_{10} \mathrm{CH}_{2}-\right]$, 1.46-1.71 (m, $\left.-\mathrm{CHCH}_{3}-\right)$, 2.76-3.36 (br, $\left.4 \mathrm{H},-\mathrm{SCH}_{2} \mathrm{CH}_{2}-\right), 3.36-3.69\left[\mathrm{br}, 4 \mathrm{H},-\mathrm{OC}(=\mathrm{O}) \mathrm{NHCH}_{2} \mathrm{CH}_{2} \mathrm{~S}-\right.$ and $-\mathrm{CH}_{2} \mathrm{NH}_{2}$ ], 4.06-4.21 (m, $\left.-\mathrm{CH}_{2} \mathrm{CH}_{2} \mathrm{OCO}-\right)$, 5.08-5.32 [m, $\left.-\mathrm{COCH}\left(\mathrm{CH}_{3}\right) \mathrm{O}-\right]$, 7.91-8.15 (br, $\left.-\mathrm{CH}_{2} \mathrm{NH}_{2}\right)$ (Figure S1).

\subsection{Synthesis of Tetraethylenepentamine/Galactose-Modified Dextran-Grafted-Disulfanyl-Ethyl-OLA (EI $/$ /Gal-Dex-g-SS-OLA)}

The synthesis of tetraethylenepentamine/galactose-modified dextran-grafted-disulfanyl-ethylOLA (EI 4 /Gal-Dex-g-SS-OLA) was carried out according to Scheme 2. Dex ( $200 \mathrm{mg}, 4.65 \mu \mathrm{mol}$, $1.23 \mathrm{mmol}$ of glucose unit) was dissolved in $3 \mathrm{~mL}$ of anhydrous dimethylsulfoxide (DMSO). CDI $(120 \mathrm{mg}, 558 \mu \mathrm{mol})$ was added, and the solution was stirred at $\mathrm{rt}$ for $1 \mathrm{~h}$ to activate the hydroxyl groups. The reaction mixture was poured into acetone and washed three times. The precipitate was dried in vacuo at $\mathrm{rt}$ to give a white powder (140 mg, yield 55\%). The activated Dex (Dex-CI) (140 mg, $2.81 \mu \mathrm{mol}, 110$ imidazole groups per macromolecule), OLA-SS- $\mathrm{NH}_{2}(96 \mathrm{mg}, 30.9 \mu \mathrm{mol})$, and DMAP (11 mg, $92.7 \mu \mathrm{mol}$ ) were dissolved in $3 \mathrm{~mL}$ of anhydrous DMSO, and stirred at $40{ }^{\circ} \mathrm{C}$ for $24 \mathrm{~h}$. Gal- $\mathrm{NH}_{2}$ ( $45 \mathrm{mg}, 112 \mu \mathrm{mol})$ and TFA-EI ${ }_{4}(70 \mathrm{mg}, 245 \mu \mathrm{mol})$ were added and the reaction mixture was stirred at $40{ }^{\circ} \mathrm{C}$ for a further $48 \mathrm{~h}$. The reaction mixture was poured into acetone and washed three times. The precipitate was dried in vacuo at $\mathrm{rt}$ to give a white powder of $\mathrm{EI}_{4} / \mathrm{Gal}-\mathrm{Dex}-\mathrm{g}$-SS-OLA $(182 \mathrm{mg}$, yield $99 \%$ (based on Dex)). Each component was characterized by ${ }^{1} \mathrm{H}$ NMR after hydrolysis using $\mathrm{NaOD} / \mathrm{D}_{2} \mathrm{O}$ (Figure S2).

${ }^{1} \mathrm{H}$ NMR $\left(400 \mathrm{MHz}, \mathrm{NaOD} / \mathrm{D}_{2} \mathrm{O}\right): \delta=1.16\left(\mathrm{~d}, \mathrm{CH}_{3} \mathrm{CH}-\right), 2.27-2.79$ (br, $\left.-\mathrm{NHCH}_{2} \mathrm{CH}_{2}-\right)$, 3.06-3.90 (m, $\mathrm{H}^{2}-\mathrm{H}^{6}$ of Dex and Gal), $3.94\left(\mathrm{q}, \mathrm{CH}_{3} \mathrm{CH}-\right), 4.32-4.43\left(\mathrm{~m}, \mathrm{H}^{1}\right.$ of $\left.\mathrm{Gal}\right), 4.71-5.13$ (br, $\mathrm{H}^{1}$ of Dex). 


\subsection{Preparation of Nanogel}

$\mathrm{EI}_{4} /$ Gal-Dex-g-SS-OLA ( $20 \mathrm{mg}$ ), dissolved in $2 \mathrm{~mL}$ of DMSO, was mixed with $8 \mathrm{~mL}$ of water with vigorous stirring at $\mathrm{rt}$. The resulting solution was placed in a dialysis tube (molecular weight cut off (MWCO): 3500), and dialyzed for $24 \mathrm{~h}$ against water at $\mathrm{rt}$ to remove organic solvent. Next, the aqueous solution was lyophilized to give a white powder of the nanogel (17 mg, yield $88 \%$ ). To prepare the nanogel solution, a predetermined amount of dried nanogel was dispersed in PB solution.

\subsection{Stability of Nanogels under Reductive Conditions}

To investigate the stability and dissociation behavior of the nanogels by cleavage of the disulfide bonds between Dex and OLA chains under reductive conditions, PB solutions of GSH or DTT $(10 \mathrm{mM})$ as reductive agents were added to the nanogel PB solutions $(1 \mathrm{mg} / \mathrm{mL})$. After certain periods, observation by the naked eye or hydrodynamic diameter measurements by DLS were carried out. Additionally, PB solutions of nanogels entrapping pyrene were prepared, and the fluorescence spectra were monitored after the addition of reductive agents.

\subsection{Cell Viability Test}

Mouse fibroblast NCTC clone 929 (L929) cells were obtained from the Health Science Research Resources Bank (HSRRB, Japan). Cell viability tests of nanogels were performed using the WST-8 assay kit against L929 cells. L929 cells $\left(100 \mu \mathrm{L}, 1 \times 10^{4}\right.$ cells $\left./ \mathrm{mL}\right)$ were grown on 96-well microplates for $24 \mathrm{~h}$ in E-MEM containing 10\% fetal bovine serum (FBS) and under a humidified atmosphere of $5 \%(v / v) \mathrm{CO}_{2}$ at $37^{\circ} \mathrm{C}$. The cells were exposed to various concentrations $\left(2.0 \times 10^{-4}\right.$ to $1.0 \times 10^{-1}$ $\mathrm{mg} / \mathrm{mL}$ ) of nanogels and control samples dissolved in phosphate-buffered saline $(-)$ [PBS( - ] for $23 \mathrm{~h}$. Thereafter, $10 \mu \mathrm{L}$ of WST-8 reagent was added to all cells and the cells were incubated for an additional $1 \mathrm{~h}$ (total $24 \mathrm{~h}$ ). At the end of the incubation period, the microplates were read at $450 \mathrm{~nm}$ in a microplate reader. The control and background were chosen at a total of $110 \mu \mathrm{L}$ of FBS-containing E-MEM with or without cells, respectively. The average background absorbance from the background wells was subtracted from the sample data. The values for each sample were in the linear region of the standard curve of the WST- 8 assay. Data are expressed as the mean and SE $(n=6)$. The cell viability was calculated using the following equation:

$$
\text { cell viability }(\%)=(\mathrm{Nt} / \mathrm{Nc}) \times 100 \text {, }
$$

where Nt and Nc are the numbers of cells with and without samples after $24 \mathrm{~h}$ of incubation, respectively. Sodium dodecyl sulfate (SDS, surfactant) was used as a positive control.

\subsection{Cellular Uptake of Nanogels into HepG2 Cells}

Cellular uptake of nanogels into human hepatocarcinoma (HepG2) cells having galactose receptors was investigated by flow cytometric analysis and confocal laser scanning microscopy (CLSM) using FITC-labeled nanogels. The introduction of FITC into Dex was carried out by mixing FITC with Dex. Dex $(4.3 \mathrm{~g}, 100 \mu \mathrm{mol})$ and FITC (39 mg, $100 \mu \mathrm{mol})$ were dissolved in anhydrous DMSO $(20 \mathrm{~mL})$ under an Ar atmosphere and stirred overnight. Acetone was added to the reaction mixture to produce a precipitate. The obtained precipitate was dissolved in water and the aqueous solution was freeze-dried to give a yellow powder of FITC-Dex ( $3.85 \mathrm{~g}$, yield 89\%). FITC-labeled Dex-g-SS-OLA was prepared using FITC-Dex by a similar method as described above. The FITC-labeled $\mathrm{EI}_{4} / \mathrm{Gal}-\mathrm{Dex}-\mathrm{g}$-SS-OLA nanogels were prepared by a dialysis method using a mixture of $\mathrm{EI}_{4} /$ Gal-Dex-g-SS-OLA and FITC-labeled Dex-g-SS-OLA (9:1). Other FITC-labeled nanogels

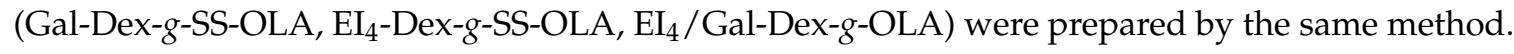

The HepG2 cell line was purchased from RIKEN Cell Bank (RCB, Wako, Japan). HepG2 cells were cultivated in D-MEM containing 10\% FBS in a humidified atmosphere of $5 \%(v / v) \mathrm{CO}_{2}$ at $37^{\circ} \mathrm{C}$. Upon observation of cellular uptake of nanogels by flow cytometric analysis, the cells were seeded on 
6-well culture dishes at $2.0 \times 10^{5}$ cells / well and incubated for $24 \mathrm{~h}$. FITC-labeled nanogels $(200 \mu \mathrm{L}, 1$ $\mathrm{mg} / \mathrm{mL})$ dissolved in PBS(-) were added to the cell culture medium and incubated for $4 \mathrm{~h}$ at $37^{\circ} \mathrm{C}$. Cells were washed with PBS(-), treated by trypsin/ethylenediaminetetraacetic acid (EDTA) PBS(-) solution, and washed another three times with PBS(-). Cells were suspended in PBS(-) and counted by using a flow cytometer (Gallios, Beckman Coulter, Inc., Brea, CA, USA) with an argon-ion coherent beam laser $(488 \mathrm{~nm})$.

For the direct observation of cellular uptake of nanogels into the cells and their localization in each cell, the HepG2 cells were seeded on $35 \mathrm{~mm}$ culture dishes at $1 \times 10^{5}$ cells and incubated for $24 \mathrm{~h}$. Lysosomes of the cells were stained by LysoTracker RED for $40 \mathrm{~min}$ at $37^{\circ} \mathrm{C}$. The cells were washed three times with PBS(-), and the medium was replaced with fresh medium. FITC-labeled nanogels $(1 \mathrm{mg} / \mathrm{mL})$ dissolved in PBS(-) were added to the culture medium of the HepG2 cells and incubated for $5 \mathrm{~min}$ and a further $5 \mathrm{~h}$ at $37^{\circ} \mathrm{C}$. The cells were washed three times with PBS(-) and observed on a confocal laser scanning microscope (CLSM) (LSM5Pascal, Carl Zeiss Co., Ltd., Jena, Germany) at the predetermined incubation times.

\section{Conclusions}

We prepared nanogels composed of Dex with OLA chains attached via disulfide bonds, galactose residues, and oligo-amine groups to accelerate collapse under reductive conditions, facilitate cellular uptake by receptor-mediated endocytosis, and escape from endosomes by the proton sponge effect. The average hydrodynamic diameter of the $\mathrm{EI}_{4} / \mathrm{Gal}-\mathrm{Dex}-\mathrm{g}-\mathrm{SS}-\mathrm{OLA}$ nanogels was $285 \mathrm{~nm}$, which decreased under reductive conditions. $\mathrm{EI}_{4} / \mathrm{Gal}-\mathrm{Dex}-\mathrm{g}$-SS-OLA nanogels had no cytotoxicity against L929 fibroblast cells. Uptake of $\mathrm{EI}_{4} / \mathrm{Gal}$-Dex-g-SS-OLA nanogels into HepG2 cells was confirmed by flow cytometric analysis and CLSM observations. The CLSM observations also suggested that $\mathrm{EI}_{4} /$ Gal-Dex-g-SS-OLA nanogels uptaken by receptor-medicated endocytosis could escape from the endosomes and subsequently collapse in the cytosol. Although we did not incorporate drugs into the nanogels in this study, $\mathrm{EI}_{4}$ /ligand-Dex-g-SS-OLA nanogels entrapping drugs can be expected to release the drugs efficiently to the cytosol during the collapse under reductive conditions, after ligand-receptor mediated endocytosis and endosomal escape. The biodegradable nanogels developed here, to control cellular traffic, should have great potential as drug delivery carriers for both hydrophobic and hydrophilic drugs.

Supplementary Materials: Supplementary materials can be found at http:/ /www.mdpi.com/1422-0067/19/6/ 1606/s1.

Author Contributions: Y.O. and A.T. designed experiments and wrote the manuscript. A.T. performed experiments. A.K. analyzed the data. Y.O. revised the manuscript. Y.O. provided overall supervision and coordinate all the experimental activities.

Funding: This work was financially supported in part by the Private University Research Branding Project: Matching Fund Subsidy from Ministry of Education, Culture, Sports, Science and Technology (MEXT) Japan (2016-2020), a Grant-in-Aid for Scientific Research (16H01854) from the Japan Society for the Promotion of Science (JSPS), and by the Kansai University Outlay Support for Establishing Research Centers, 2016.

Acknowledgments: The authors thank Yasuhiko Iwasaki, Kansai University for measurements on DLS and flow cytometry.

Conflicts of Interest: The authors declare no conflicts of interest.

\section{References}

1. Yuki, Y.; Nochi, T.; Kong, I.G.; Takahashi, H.; Sawada, S.; Akiyoshi, K.; Kiyono, H. Nanogel-based antigen-delivery system for nasal vaccines. Biotechnol. Genet. Eng. Rev. 2013, 29, 61-72. [CrossRef] [PubMed]

2. Morimoto, N.; Hasegawa, U.; Sugawara, A.; Yamane, S.; Akiyoshi, K. Polysaccharide nanogel engineering: Design of nano-structured hydrogel materials and application to biotechnology and medicine. In Nanotechnololgy in Carbohydrate Chemistry; Yuasa, H., Ed.; Transworld Research Network: Keraka, India, 2006; pp. 67-87. 
3. Sasaki, Y.; Akiyoshi, K. Nanogel engineering for new nanobiomaterials: From chaperoning Engineering to biomedical applications. Chem. Rec. 2010, 10, 366-376. [CrossRef] [PubMed]

4. Ayame, H.; Morimoto, N.; Akiyoshi, K. Self-Assembled cationic nanogels for intracellular protein delivery. Bioconjug. Chem. 2008, 19, 882-890. [CrossRef] [PubMed]

5. Eckmann, D.M.; Composto, R.J.; Tsourkasc, A.; Muzykantov, V.R. Nanogel carrier design for targeted drug delivery. J. Mater. Chem. B 2014, 2, 8085-8097. [CrossRef] [PubMed]

6. Nuhn, L.; Tomcin, S.; Miyata, K.; Mailänder, V.; Landfester, K.; Kataoka, K.; Zentel, R. Size-dependent knockdown potential of siRNA-loaded cationic nanohydrogel particles. Biomacromolecules 2014, 15, 4111-4121. [CrossRef] [PubMed]

7. Muraoka, D.; Harada, N.; Hayashi, T.; Tahara, Y.; Momose, F.; Sawada, S.; Mukai, S.; Akiyoshi, K.; Shiku, H. Nanogel-based immunologically stealth vaccine targets macrophages in the medulla of lymph node and induces potent antitumor immunity. ACS Nano 2014, 8, 9209-9218. [CrossRef] [PubMed]

8. Chiang, W.H.; Huang, W.C.; Shen, M.Y.; Wang, C.H.; Huang, Y.F.; Lin, S.C.; Chern, C.S.; Chiu, H.C. Dual-layered nanogel-coated hollow lipid/polypeptide conjugate assemblies for potential $\mathrm{pH}$-triggered intracellular drug release. PLoS ONE 2014, 9, e92268. [CrossRef] [PubMed]

9. Morimoto, N.; Hirano, S.; Takahashi, H.; Loethen, S.; Thompson, D.H.; Akiyoshi, K. Self-assembled $\mathrm{pH}$-sensitive cholesteryl pullulan nanogel as a protein delivery vehicle. Biomacromolecules 2013, 14, 56-63. [CrossRef] [PubMed]

10. Averick, S.E.; Paredes, E.; Irastorza, A.; Shrivats, A.R.; Srinivasan, A.; Siegwart, D.J.; Magenau, A.J.; Cho, H.Y.; Hsu, E.; Averick, A.A.; et al. Preparation of cationic nanogels for nucleic acid delivery. Biomacromolecules 2012, 13, 3445-3449. [CrossRef] [PubMed]

11. Molla, M.R.; Marcinko, T.; Prasad, P.; Deming, D.; Garman, S.C.; Thayumanavan, S. Unlocking a caged lysosomal protein from a polymeric nanogel with a $\mathrm{pH}$ trigger. Biomacromolecules 2014, 15, 4046-4053. [CrossRef] [PubMed]

12. Wei, X.; Senanayake, T.H.; Bohling, A.; Vinogradov, S.V. Targeted nanogel conjugate for improved stability and cellular permeability of curcumin: Synthesis, pharmacokinetics, and tumor growth inhibition. Mol. Pharmaceutics 2014, 11, 3112-3122. [CrossRef] [PubMed]

13. Xing, T.; Mao, C.; Lai, B.; Yan, L. Synthesis of disulfide-cross-linked polypeptide nanogel conjugated with a near-infrared fluorescence probe for direct imaging of reduction-induced drug release. ACS Appl. Mater. Interfaces 2012, 4, 5662-5672. [CrossRef] [PubMed]

14. Akiyoshi, K.; Deguchi, S.; Tajima, H.; Nishikawa, T.; Sunamoto, J. Microscopic structure and thermoresponsiveness of a hydrogel nanoparticle by self-assembly of a hydrophobized polysaccharide. Macromolecules 1997, 30, 857-861. [CrossRef]

15. Nagahama, K.; Mori, Y.; Ohya, Y.; Ouchi, T. Biodegradable nanogel formation of polylactide-grafted dextran copolymer in dilute aqueous solution and enhancement of its stability by stereocomplexation. Biomacromolecules 2007, 8, 2135-2141. [CrossRef] [PubMed]

16. Nagahama, K.; Ouchi, T.; Ohya, Y. Biodegradable nanogels prepared by self-assembly of poly(L-lactide)-grafted dextran: Entrapment and release of protein. Macromol. Biosci. 2008, 8, 1044-1052. [CrossRef] [PubMed]

17. Bae, Y.; Kataoka, K. Intelligent polymeric micelles from functional poly(ethylene glycol)-poly(amino acid) block copolymers. Adv. Drug Deliv. Rev. 2009, 61, 768-784. [CrossRef] [PubMed]

18. Maeda, H.; Matsumura, Y. Tumoritropic and lymphotropic principles of macromolecular drugs. Crit. Rev. Ther. Drugs Carr. Syst. 1989, 6, 193-210.

19. Kusumoto, K.; Akita, H.; Ishitsuka, T.; Matsumoto, Y.; Nomoto, T.; Furukawa, R.; El-Sayed, A.; Hatakeyama, H.; Kajimoto, K.; Yamada, Y.; et al. Lipid envelope-type nanoparticle incorporating a multifunctional peptide for systemic siRNA delivery to the pulmonary endothelium. ACS Nano 2013, 7, 7534-7541. [CrossRef] [PubMed]

20. Tekade, R.K.; Kumar, P.V.; Jain, N.K. Dendrimers in oncology: An expanding horizon. Chem. Rev. 2009, 109, 49-87. [CrossRef] [PubMed]

21. Howard, M.; Zern, B.J.; Anselmo, A.C.; Shuvaev, V.V.; Mitragotri, S.; Muzykantov, V. Vascular targeting of nanocarriers: Perplexing aspects of the seemingly straightforward paradigm. ACS Nano 2014, 8, 4100-4132. [CrossRef] [PubMed] 
22. Trail, P.A.; Willner, D.; Lasch, S.J.; Henderson, A.J.; Hofstead, S.; Casazza, A.M.; Firestone, R.A.; Hellstrom, I.; Hellstrom, K.E. Cure of xenografted human carcinomas by BR96-doxorubicin immunoconjugates. Science 1993, 261, 212-215. [CrossRef] [PubMed]

23. Jaracz, S.; Chen, J.; Kuznetsova, L.V.; Ojima, I. Recent advances in tumor-targeting anticancer drug conjugates. Bioorg. Med. Chem. 2005, 13, 5043-5054. [CrossRef] [PubMed]

24. Boussif, O.; Lezoualch, F.; Zanta, M.A.; Mergny, M.D.; Scherman, D.; Demeneix, B.; Behr, J.P. A versatile vector for gene and oligonucleotide transfer into cells in culture and in vivo: Polyethylenimine. Proc. Natl. Acad. Sci. USA 1995, 92, 7297-7301. [CrossRef] [PubMed]

25. Jäger, M.; Schubert, S.; Ochrimenko, S.; Fischer, D.; Schubert, U.S. Branched and linear poly(ethylene imine)-based conjugates: Synthetic modification, characterization, and application. Chem. Soc. Rev. 2012, 41, 4755-4767. [CrossRef] [PubMed]

26. Midoux, P.; Pichon, C.; Yaouanc, J.J.; Jaffrès, P.A. Chemical vectors for gene delivery: A current review on polymers, peptides and lipids containing histidine or imidazole as nucleic acids carriers. Br. J. Pharmacol. 2009, 157, 166-178. [CrossRef] [PubMed]

27. Ko, N.R.; Oh, J.K. Glutathione-triggered disassembly of dual disulfide located degradable nanocarriers of polylactide-based block copolymers for rapid drug release. Biomacromolecules 2014, 15, 3180-3189. [CrossRef] [PubMed]

28. Li, Y.; Lei, X.; Dong, H.; Ren, T. Sheddable, degradable, cationic micelles enabling drug and gene delivery. RSC Adv. 2014, 4, 8165-8176. [CrossRef]

29. Tang, L.Y.; Wang, Y.C.; Li, Y.; Du, J.Z.; Wang, J. Shell-detachable micelles based on disulfide-linked block copolymer as potential carrier for intracellular drug delivery. Bioconjug. Chem. 2009, 20, 1095-1099. [CrossRef] [PubMed]

30. Lis, H.; Sharon, N. Lectins: Carbohydrate-specific proteins that mediate cellular recognition. Chem. Rev. 1998, 98, 637-674. [CrossRef] [PubMed]

31. Gupta, A.; Gupta, R.K.; Gupta, G.S. Targeting cells for drug and gene delivery: Emerging application of mannans and mannan binding lectines. J. Sci. Ind. Res. 2009, 68, 465-483.

32. Tsuji, H.; Sato, S.; Masaki, N.; Arakawa, Y.; Kuzuya, A.; Ohya, Y. Stereocomplex crystallization and homo-crystallization of enantiomeric poly(lactic acid-co-alanine)s with ester and amide linkages. Polym. Chem. 2018, 9, 565-575. [CrossRef]

33. Murata, J.; Ohya, Y.; Ouchi, T. Possibility of application of quaternary chitosan having pendant galactose residues as gene delivery tool. Carbohydr. Polym. 1996, 29, 69-74. [CrossRef]

34. Niu, J.; Liu, Z.; Fu, L.; Shi, F.; Ma, H.; Ozaki, Y.; Zhang, X. Surface-imprinted nanostructured layer-by-layer film for molecular recognition of theophylline derivatives. Langmuir 2008, 24, 11988-11994. [CrossRef] [PubMed]

35. Konig, S.G.; Kramer, R. Polyamine-modified near-infrared cyanine dyes for targeting the nuclei and nucleoli of cells. Dyes Pigments 2017, 145, 80-94. [CrossRef]

(c) 2018 by the authors. Licensee MDPI, Basel, Switzerland. This article is an open access article distributed under the terms and conditions of the Creative Commons Attribution (CC BY) license (http://creativecommons.org/licenses/by/4.0/). 